\title{
In die Rente wider Willen?
}

Ältere Beziehende von Arbeitslosengeld II (ALG II) können vonseiten der Jobcenter dazu verpflichtet werden, eine Altersrente vorzeitig, also auch um den Preis von Abschlägen, zu beantragen. Unter anderem wegen dieses möglichen Zwanges und der damit verbundenen Fremdbestimmung der ALG-II-Beziehenden durch die Jobcenter, aber auch wegen der Fehlanreize, die sich mit Blick auf die Jobcenter aus dieser Regelung ergeben, steht die Pflicht zur vorzeitigen Inanspruchnahme einer Altersrente in der Kritik. Dieser Beitrag untersucht die Auswirkungen dieser Regelung für den Zugang in Altersrente von ALG-IIBeziehenden. Die Ergebnisse stützen die Vermutung, dass sich das Rentenzugangsverhalten der ALG-II-Beziehenden nicht grundlegend ändern würde, wenn die Pflicht zur vorzeitigen Inanspruchnahme einer Altersrente abgeschafft werden würde.

MARTIN BRUSSIG

\section{Die Pflicht zur Inanspruchnahme einer Altersrente}

\subsection{Gesetzliche Grundlagen}

Ältere Arbeitslose, die Leistungen nach dem Sozialgesetzbuch, Zweites Buch (SGB II) beziehen, sind verpflichtet, eine Altersrente zu beantragen, um damit den Bezug von ALG II zu beenden ( $\$ 5$ SGB II). Dies schließt auch die Inanspruchnahme einer um Abschläge geminderten Altersrente ein. Diese Verpflichtung ergibt sich aus der „Nachrangigkeit" des ALG II, also aus dem Grundsatz, dass die Grundsicherung für erwerbsfähige Hilfebedürftige erst nach dem Ausschöpfen aller anderen Möglichkeiten, die materielle Existenzgrundlage zu sichern, gewährt wird. $\mathrm{Zu}$ den vorrangig zu nutzenden Einkommen zählen auch Altersrenten. Kommt die leistungsberechtigte Person dieser Verpflichtung nicht nach, ist das Jobcenter als Leistungsträger berechtigt, für diese Person den Rentenantrag zu stellen.

Die Verpflichtung zur Inanspruchnahme von Altersrenten besteht seit Inkrafttreten des SGB II zum 01.01.2005 und ist seitdem mehrfach präzisiert worden. Durch eine erste Ergänzung wurde bestimmt, dass der Leistungsträger nicht nur anstelle der ALG II beziehenden Person den Rentenantrag stellen, sondern auch Widerspruch gegen die Entscheidung der Rentenversicherung einlegen kann (wirk- sam ab 01.08.2006). Noch wichtiger jedoch war die Konkretisierung, eine vorzeitig beziehbare Altersrente nicht vor Vollendung des 63. Lebensjahres in Anspruch nehmen zu müssen ( $\$ 12 \mathrm{a}$, SGB II wirksam ab 01.01.2008). Mit dieser Präzisierung hat der Gesetzgeber eine Angleichung der Altersgrenze an die Altersrente wegen Arbeitslosigkeit erreicht, deren frühestmögliche Inanspruchnahme von 60 auf 63 Jahre angehoben wurde. Ebenfalls zum 01.01.2008 hat der Gesetzgeber eine Unbilligkeitsverordnung in Kraft gesetzt, der zufolge eine vorzeitige Inanspruchnahme einer Altersrente insbesondere dann nicht erwirkt wird, wenn ALG II nur aufstockend neben einer bestehenden Erwerbstätigkeit oder laufendem Arbeitslosengeld bezogen wird, wenn eine Erwerbsaufnahme unmittelbar bevorsteht oder in nächster Zukunft eine abschlagsfreie Altersrente bezogen werden kann.

In den ersten Jahren des SGB II ist die Verpflichtung zur vorzeitigen Inanspruchnahme einer Altersrente im Zusammenhang mit der sogenannten 58er-Regelung zu sehen ( $\$ 428$ SGB III a.F.). Nach dieser Regelung war ein Bezug von Arbeitslosengeld ohne Verpflichtung zur Arbeitssuche möglich, sofern sich die betreffende Person verpflichtete, zum frühestmöglichen Zeitpunkt eine abschlagsfreie Altersrente zu beantragen. Dieser „Leistungsbezug unter erleichterten Voraussetzungen “ hatte seinen Ursprung in einer Zeit, in der Arbeitslosengeld für Ältere bis zu 32 Monate gezahlt wurde und ein abschlagsfreier Rentenzugang in die Altersrente wegen Arbeitslosigkeit ab 60 Jahren mög- 
lich war. Die Möglichkeit zum erleichterten Leistungsbezug stand mit Einführung des SGB II auch für ALG-II-Beziehende zur Verfügung. Für diese Gruppe hatte er den wesentlichen Effekt, dass der abschlagsfreie Rentenzugangszeitpunkt geschützt war, der aufgrund der Heraufsetzung der abschlagsfreien Altersgrenzen längst nicht mehr bei 60 Jahren, sondern (seit 2007) bei 65 Jahren lag (vgl. Brussig/Wübbeke 2009; Wübbeke 2013). Bis zur Schließung des erleichterten Leistungsbezuges für Neuzugänge (zum 31.12.2009) konnte die Verpflichtung zum vorzeitigen Rentenbezug also vermieden werden. Die Jobcenter behandeln ALG-II-Beziehende, die sich auf diese Möglichkeit hätten stützen können, so, als ob sie dies auch tatsächlich getan hätten. Es gilt also ein Bestandsschutz.

\subsection{Sozialrechtliche Einordnung}

Die Nachrangigkeit der Grundsicherung für erwerbsfähige Hilfebedürftige gegenüber anderen Sozialleistungen, wie der Altersrente, und die Pflicht, andere Sozialleistungen zu beantragen und gegebenenfalls auf diese auszuweichen, sind im deutschen Sozialrecht weder neu noch einzigartig. Zwar galt die Arbeitslosenhilfe (und gilt erst recht das Arbeitslosengeld) im Regelfall nicht als nachrangig. Doch die Sozialhilfe, das ursprünglich neben der Arbeitslosenhilfe andere Leistungssystem, aus deren beider Verschmelzung die Grundsicherung für erwerbsfähige Hilfebedürftige erwuchs, war bereits eine nachrangige Sozialleistung: „Sozialhilfe erhält nicht, wer sich selbst helfen kann oder wer die erforderliche Hilfe von anderen, besonders von Angehörigen oder von Trägern anderer Sozialleistungen, erhält" ( $\$ 2$ BSHG).

Das individuelle „Dispositionsrecht“, also die individuelle Entscheidung darüber, eine Sozialleistung zu beantragen, ist nicht nur in den bedarfsgeprüften und steuerfinanzierten sozialen Sicherungssystemen eingeschränkt, sondern auch in der Sozialversicherung. Es ist beispielsweise für Personen eingeschränkt, die Arbeitslosengeld nur deshalb erhalten, weil ihr Anspruch auf Krankengeld ausgelaufen ist, ohne dass ihre Erwerbsfähigkeit wiederhergestellt ist $(\$ 145$ SGB III). Sie sind dazu verpflichtet, Leistungen zur medizinischen Rehabilitation (Reha) oder zur beruflichen Wiedereingliederung bei der Rentenversicherung zu beantragen. Dies bedeutet unter Umständen - nämlich wenn prognostiziert wird, dass auch durch die Rehabilitation die Erwerbsfähigkeit nicht zurückgewonnen wird -, dass der Reha-Antrag in einen Rentenantrag umgedeutet und eine Erwerbsminderungsrente bewilligt wird. Kommt der Versicherte dieser Aufforderung nicht nach, kann die Agentur für Arbeit die Zahlung von Arbeitslosengeld einstellen. Auch Krankenkassen können Versicherte schon während des Krankengeldbezuges zu einer Reha-Antragstellung auffordern, an deren Ende wiederum eine Erwerbsminderungsrente stehen kann; und sie können, wenn der Versicherte seine Kooperation verweigert, versuchen, seine Mitwirkung durch Einstellung des Krankengeldes zu erzwingen. Die Möglichkeit zur verpflichtenden Antragstel- lung lässt sich damit rechtfertigen, dass Personen dem tatsächlich zuständigen Sicherungssystem zugeordnet werden. Dabei geht es nicht nur darum, den Grund des Einkommensausfalls korrekt zu erfassen (beispielsweise aufgrund vorübergehender Arbeitsunfähigkeit oder dauerhafter Erwerbsunfähigkeit) und den zuständigen Leistungsträger zu bestimmen, sondern auch darum, dass der zuständige Leistungsträger auch Dienstleistungen bereithält bzw. finanziert, die auf das jeweilige Risiko abgestimmt sind. Gleichwohl bedeutet die Beschränkung der Dispositionsfreiheit des Versicherten einen erheblichen Eingriff in seine Selbstbestimmung. Auch bei der Aufforderung zu einem Reha-Antrag - der ohne Eingriffsmöglichkeit des Versicherten in einen Rentenantrag umgewandelt werden kann - gibt es deshalb ähnlich wie bei der Unbilligkeitsverordnung (s. o.) Schranken für die Träger. So darf der Versicherte es unterlassen, der Aufforderung der Krankenkasse nachzukommen, wenn noch eine erhebliche Verbesserung des Rentenanspruchs erreicht werden kann. Ein Verweigerungsrecht hat er auch, wenn aufgrund tarifvertraglicher Regelungen ein Rentenantrag automatisch zum Arbeitsplatzverlust führen würde und Ansprüche auf Betriebsrente durch einen frühzeitigen Rentenbeginn verloren gehen würden.

\section{Kritische Einwände und Diskussionsbedarf}

Weder die Nachrangigkeit von ALG II noch die Einschränkung des Dispositionsrechts sind also neu oder einzigartig. Dennoch ist gerade die Pflicht zur Inanspruchnahme einer vorgezogenen Altersrente - stärker als die anderen hier aufgeführten Beispiele - in der Kritik. Sie lässt sich in vier Punkten festmachen:

(1) Der Bezug einer Altersrente ist irreversibel. Sie kann nicht ausgesetzt, durch weitere Erwerbstätigkeit aufgebessert und zu einem späteren Zeitpunkt erneut beantragt und bezogen werden. Zudem wird der Status als Altersrentner fremdbestimmt und dauerhaft zugewiesen. Dies ist ein wesentlicher Unterschied zur Erwerbsminderungsrente, die grundsätzlich befristet zu bewilligen ist und den Status als erwerbsgemindert nicht lebenslang festschreibt. Einzigartig im Vergleich zu den geschilderten Fällen in der Arbeitslosen- und Krankenversicherung ist die Möglichkeit des Grundsicherungsträgers zur stellvertretenden Antragstellung, die der betroffenen Person die Möglichkeit nimmt, über ihren sozialrechtlichen Status selbst zu bestimmen.

(2) Bei einer vorzeitigen Inanspruchnahme von Altersrenten mindern versicherungsmathematische Abschläge den Rentenzahlbetrag, zumal - zumindest bis Ende 2010 - für ALG-II-Beziehende rentensteigernde Beiträge in der Rentenversicherung abgeführt wurden (Mika et al. 2014). Ab- 
gesehen von diesem fremdbestimmten Eingriff in den bereits erworbenen Rentenanspruch kann im Extremfall dadurch eine Bedürftigkeit im Rentenalter erst entstehen, die andernfalls hätte vermieden werden können. Die Vermeidung eines dauerhaften Bezugs der Grundsicherung im Alter, die bei einem abschlagsfreien Rentenbeginn nicht eintreten würde, wird in der Unbilligkeitsverordnung nicht als unbillige Härte benannt.

(3) Für die betroffenen Personen entsteht möglicherweise eine Lücke oder zumindest ein Nachteil, um das Existenzminimum zu sichern. Denn wenn die vorzeitig bezogene Rente (unter Einschluss aller weiteren Einkommen) nicht bedarfsdeckend ist, können weder die Grundsicherung für erwerbsfähige Hilfebedürftige (also weiterhin aufstockendes ALG II) noch die Grundsicherung für Ältere bezogen werden: Die Grundsicherung für erwerbsfähige Hilfebedürftige scheidet aus, weil ihre Beendigung das Ziel für den Rentenbezug war, und die Grundsicherung im Alter greift nicht, weil sie an die Vollendung der Regelaltersgrenze geknüpft ist. Infrage kommt dann am ehesten die Hilfe zum Lebensunterhalt („Sozialhilfe“, Drittes Kapitel SGB XII), bei der allerdings die Bedingungen des Leistungsbezuges restriktiver sind als in der Grundsicherung für Erwerbsfähige (SGB II) bzw. im Alter (Viertes Kapitel SGB XII), denn das Schonvermögen ist niedriger und der Unterhaltsrückgriff auf das Einkommen der Kinder setzt eher ein.

(4) Es besteht schließlich die Gefahr, dass die Pflicht zur vorzeitigen Inanspruchnahme einer Altersrente falsche Anreize für die Jobcenter setzt. Entgegen den positiven Erfahrungen bei der Vermittlung älterer Langzeitarbeitsloser im Bundesprogramm Perspektive 50plus sind sie nämlich im Fall der verpflichtenden Rentenantragstellung zu einer systematischen Aussteuerung von Älteren statt zu ihrer Aktivierung angehalten. Angesichts vorliegender Berichte über die Arbeitsbelastungen in Jobcentern aufgrund hoher Fallzahlen (zuletzt SOFI et al. 2013) wäre ein solches Verhalten der Jobcenter nicht nur gesetzeskonform, sondern für sie selbst auch arbeitserleichternd.

Für eine Beurteilung der Pflicht zur Inanspruchnahme einer Altersrente reicht eine rechtliche Betrachtung des Für und Wider der Einschränkungen des individuellen Selbstbestimmungsrechts nicht aus. Notwendig ist darüber hinaus empirisches Wissen über die tatsächliche Verbreitung und die Wirkungen dieser Bestimmung. Doch weder zur Verbreitung noch zu den Folgen der Pflicht zur Inanspruchnahme einer Altersrente aus dem ALG-II-Bezug liegen gesicherte Informationen vor. Dies ist wesentlich, wie unten weiter ausgeführt wird, durch fehlende Datengrundlagen bedingt. Auch dieser Beitrag kann keine Daten zu Verbreitung, Abläufen und Motiven der Inanspruchnahme vorzeitig beziehbarer Altersrenten nutzen. Gestützt auf Sozialdaten der Rentenversicherung wird jedoch eine Annäherung an die Frage versucht, wie groß der betroffene Personenkreis ist und wie realistisch die Gefahr ist, aufgrund der Rentenabschläge auch im Alter bedürftig zu sein. Weiterhin wird diskutiert, ob Anzeichen dafür erkennbar sind, dass ALGII-Beziehende wegen der Pflicht zur Inanspruchnahme einer Altersrente ihre Rente beziehen, oder ob sie diese vielleicht nicht auch selbstmotiviert beantragen. Zunächst soll jedoch auf das vorliegende Wissen über die verpflichtende Rentenantragstellung eingegangen werden (Abschnitt 3), bevor die empirische Analyse (4) beginnt. Eine kurze Diskussion der Ergebnisse (5) beschließt diesen Aufsatz.

\section{Stand des Wissens: drei parlamen- tarische Anfragen der Linksfraktion}

Die Bundestagsfraktion der Linkspartei (Linksfraktion) hat 2009, 2011 und 2013 jeweils eine Kleine Anfrage an die Bundesregierung gestellt, die jedesmal die Pflicht zur Inanspruchnahme einer Altersrente zum Gegenstand hatte. Kleine Anfragen sind eine auf wenige Punkte beschränkte Fragestellung eines Bundestagsabgeordneten an die Bundesregierung. Sie sind ein Instrument der parlamentarischen Kontrolle (Schmidt 2007) und sollen von der Bundesregierung innerhalb von 14 Tagen schriftlich beantwortet werden (Geschäftsordnung des Deutschen Bundestages, $₫ 104$ ).

Bereits die erste der drei Anfragen verlangte eine Auskunft darüber, „wie viele Hilfebedürftige [...] seit Jahresbeginn 2008 von den Trägern des SGB II auf einen vorzeitigen Rentenbezug [...] verwiesen worden“ sind (BT-Drucksache 16/13346, S. 4). Die Antwort lautete: „Die Bundesregierung verfügt insoweit über kein verlässliches statistisches Datenmaterial“ (ebd.). Auch in der zweiten Anfrage lautete die Antwort auf eine ähnliche Frage: „Für die gefragten Angaben liegen keine Daten vor“ (BT-Drucksache 17/5410, S. 12). Und auch die dritte Anfrage, die deutlich umfangreicher ausfiel und sich ausschließlich mit der „Zwangsverrentung" beschäftigte, erkundigte sich danach, wie oft die Aufforderung für eine Rentenantragstellung in einer Eingliederungsvereinbarung vereinbart wurde (Frage 23), wie oft Leistungsberechtigte zu einer Rentenantragstellung aufgefordert wurden (Frage 24), und wie oft der Leistungsträger anstelle der leistungsberechtigten Person einen Rentenantrag gestellt hat (Frage 25). Sie wurden summarisch beantwortet: „Der Bundesregierung liegen hierzu keine Angaben vor" (BT-Drucksache 18/152). Gleichzeitig teilte die Bundesregierung mit, dass die entsprechenden Angaben in den Informationssystemen der Jobcenter (A2LL bzw. VerBIS) vorliegen, da sie Bestandteil der Kommunikation mit den Leistungsberechtigten sind. Sie werden aber nicht zentral zusammengeführt, weil sie nicht zu dem - gesetzlich vorgeschriebenen - Bereich von Daten gehören, die der Bundesagentur für Arbeit zu übermitteln sind (ebd., S. 7). Weiterhin wurden Fragen nach den Abgängen von 63-Jährigen aus dem SGB-II-Leistungsbezug gestellt. Auf 
TABELLE 1

Abgang erwerbsfähiger Leistungsberechtigter aus dem SGB-II-Leistungsbezug nach Alter

Angaben in absoluten Zahlen

\begin{tabular}{l|r|r|r|r|r|r|}
\hline Alter & Juli $\mathbf{2 0 0 8}$ & Juli $\mathbf{2 0 0 9}$ & Juli $\mathbf{2 0 1 0}$ & Juli $\mathbf{2 0 1 1}$ & Juli 2012 & Juli $\mathbf{2 0 1 3}$ \\
\hline 63 & $\mathbf{7 9 8}$ & 873 & 1.106 & 1.258 & 1.686 & 2.338 \\
\hline 64 & 2.591 & 2.787 & 2.728 & 1.987 & 760 & 855 \\
\hline 65 & 0 & 0 & 0 & 1.637 & 3.407 & 3.552 \\
\hline
\end{tabular}

Quelle: BT-Drucksache 18/152, Tabelle 6, S. 39

diese Weise wurde von den Fragestellern versucht, zu bestimmen, wie viele Personen auf die Altersrente verwiesen wurden. In der Tat ist deren Zahl mit dem Auslaufen der 58er-Regelung zum 31.12.2009, die vor der verpflichtenden vorzeitigen Rente schützte, deutlich angestiegen (Tabelle 1). Aus diesen Monatsangaben werden hochgerechnet für das Jahr 2013 bis zu 28.000 Personen ermittelt, die vermutlich wegen der verpflichtenden Inanspruchnahme in Altersrente gewechselt sind (Birkwald 2013, S. 1). ${ }^{1}$ Bezogen auf die Jahre 2011 und 2012 wären es ca. 15.000 bzw. 20.000 Personen.

Das Alter ist aber kein geeigneter Indikator für den interessierenden Sachverhalt der vorzeitig (also mit Abschlägen) beziehbaren Altersrente, denn es sind einerseits abschlagsfreie Rentenzugänge mit 63 Jahren möglich (Schwerbehinderte bestimmter Jahrgänge mit Vertrauensschutz), während andererseits einige Personen die rentenrechtlichen Bedingungen für eine vorzeitige Altersrente erst nach der Vollendung des 63. Lebensjahres erfüllen können und mithin bei den 64-Jäh- rigen zu suchen wären, deren Abgänge aus dem SGB-II-Leistungsbezug aber zurückgehen. Auffällig ist zudem, dass unter den drei ausgewiesenen Altersgruppen in den Jahren 2012 und 2013 die Mehrheit erst mit der Regelaltersgrenze in die Altersrente gewechselt ist. ${ }^{3}$ Das Ausmaß der Inanspruchnahme einer vorzeitigen Altersrente und die Folgen für eine spätere Fürsorgebedürftigkeit lassen sich so nicht abschätzen.

1 Hiervon abzuziehen sind die Personen, die wegen einer Beschäftigungsaufnahme oder aus anderen Gründen den ALG-II-Bezug verlassen haben (ebd.).

2 Der Rechtsstand bezieht sich auf 2011, weil sich die folgende empirische Analyse auf dieses Jahr bezieht.

3 Schwer zu erklären ist zudem, dass es in den Jahren 2008 bis 2010 angeblich überhaupt keine Abgänge aus dem ALG II mit 65 Jahren gegeben haben soll.

ÜBERSICHT 1

\section{Altersrenten und ihre Voraussetzungen}

\begin{tabular}{|c|c|c|c|c|}
\hline \multirow[t]{2}{*}{ Rentenart } & \multirow[t]{2}{*}{ Voraussetzung } & \multicolumn{2}{|c|}{ Zugangsalter } & \multirow[t]{2}{*}{ Kommentar } \\
\hline & & frühestmöglich & abschlagsfrei & \\
\hline Regelaltersrente & Fünf Jahre Beitragszeiten & - & 65 & Ab 2012 Anhebung auf 67 Jahre \\
\hline $\begin{array}{l}\text { Altersrente für langjährig } \\
\text { Versicherte }\end{array}$ & 35 Jahre Beitragszeiten & 63 & 65 & $\begin{array}{l}\text { Bei Vertrauensschutz abschlagsfrei ab 60, } \\
\text { ab } 2012 \text { Anhebung auf } 67 \text { Jahre }\end{array}$ \\
\hline Altersrente für Frauen & $\begin{array}{l}15 \text { Jahre Beitragszeiten, davon mind. zehn } \\
\text { nach dem } 40 \text {. Geburtstag }\end{array}$ & 60 & 65 & $\begin{array}{l}\text { Nicht für Personen des Jahrgangs } 1952 \\
\text { oder jünger }\end{array}$ \\
\hline $\begin{array}{l}\text { Altersrente nach } \\
\text { Arbeitslosigkeit }\end{array}$ & $\begin{array}{l}15 \text { Jahre Beitragszeiten, davon mind. acht in } \\
\text { den letzten zehn Jahren vor Renten- } \\
\text { beginn, mindestens ein Jahr arbeitslos } \\
\text { nach Alter } 58,5 \text { Jahre }\end{array}$ & 63 & 65 & $\begin{array}{l}\text { Nicht für Personen des Jahrgangs } 1952 \\
\text { oder jünger }\end{array}$ \\
\hline $\begin{array}{l}\text { Altersrente wegen } \\
\text { Altersteilzeitarbeit }\end{array}$ & $\begin{array}{l}\text { Wie in der Zeile zuvor, aber statt Arbeitslo- } \\
\text { sigkeit mindestens zwei Jahre Altersteilzeit- } \\
\text { arbeit direkt vor Rentenbeginn }\end{array}$ & 63 & 65 & $\begin{array}{l}\text { Nicht für Personen des Jahrgangs } 1952 \\
\text { oder jünger }\end{array}$ \\
\hline $\begin{array}{l}\text { Altersrente für } \\
\text { schwerbehinderte Menschen }\end{array}$ & $\begin{array}{l}35 \text { Jahre Beitragszeiten und anerkannte } \\
\text { Schwerbehinderung }\end{array}$ & 60 & 63 & $\begin{array}{l}\text { Bei Vertrauensschutz abschlagsfrei ab 60, } \\
\text { ab } 2012 \text { Anhebung auf } 62 \text { bzw. } 65 \text { Jahre }\end{array}$ \\
\hline
\end{tabular}




\section{Empirische Analysen}

\subsection{Vorüberlegungen und Analysestrategie}

Für die empirische Analyse ist es hilfreich, die grundsätzlichen Abläufe der Jobcenter im Umgang mit rentenberechtigten ALG-II-Beziehenden zu kennen. Sie sind in sogenannten „Fachlichen Hinweisen der Bundesagentur für Arbeit" niedergelegt, die öffentlich zugänglich sind und die die Gesetzesvorgaben in konkretes organisationales Handeln umformen (Abbildung 1).

Demnach beginnt die Rentenprüfung bereits mit der Vollendung des 60. Lebensjahres. SGB-II-Leistungsbeziehende werden vom Jobcenter aufgefordert, eine aktuelle Renteninformation der Rentenversicherung vorzulegen. Die Renteninformation enthält Angaben, ob die versicherungsrechtlichen Voraussetzungen für den Bezug einer vorzeitig beziehbaren Altersrente erfüllt sind. Damit eine der vorzeitig beziehbaren Altersrenten genutzt werden kann, müssen Vorversicherungszeiten erfüllt sein (Übersicht 1).

Das Jobcenter prüft zunächst, ob die Voraussetzungen für eine ungeminderte Altersrente bestehen. Dies ist auch unterhalb der Regelaltersgrenze möglich, z. B. für Schwerbehinderte, die die Voraussetzungen der Altersrente für schwerbehinderte Menschen erfüllen, oder für Personen, die aufgrund von Vertrauensschutzregelungen abschlagsfrei vorzeitig in Rente gehen können. Ist das der Fall, ergeht sofort und ohne weitere Prüfung eine Aufforderung zur Rentenantragstellung. Besteht hingegen ein Anspruch auf eine geminderte Altersrente, wird geprüft, ob ein Bestandsschutz (im Wesentlichen der oben besprochene „erleichterte Leistungsbezug" nach der alten 58er-Regelung) vorliegt oder unbillige Härten (s.o.) gegen eine Rentenantragstellung sprechen. Soweit das nicht der Fall ist, ergeht die Aufforderung zu einer Rentenantragstellung, sofern dies nicht bereits geschehen ist.

Es ist anzunehmen, dass „rentennahe“ SGB-II-Leistungsbeziehende zu Gesprächen in das Jobcenter einbestellt werden, in denen die Pflicht zur vorzeitigen Inanspruchnahme einer Altersrente erläutert und das weitere Vorgehen beschrieben werden. Möglicherweise findet eine Art „Rentenberatung“ statt, in der der erwartbare Rentenzahlbetrag mit dem aktuellen ALG II verglichen wird und in der vermittels einer „Vorteilsübersetzung“ (ein im Jobcenter gebräuchlicher Begriff, um Pflichten und Interessen des „Kunden“ in Einklang zu bringen) die Attraktivität selbst einer um Abschläge geminderten Rente verdeutlicht wird. Da eine uneingeschränkte Pflicht besteht, ungeminderte Altersrenten auch vor Vollendung des 63. Lebensjahres in Anspruch zu nehmen, ist davon auszugehen, dass die Rentenberatung bereits vor dem 63. Lebensjahr einsetzt. Es ist zu vermuten, dass diese Gespräche von Vermittler zu Vermittler und vielleicht sogar von Jobcenter zu Jobcenter verschieden sind. Unabhängig hiervon ist davon auszugehen,

\section{Ablaufschema „Verweis auf die Inanspruchnahme einer Altersrente”}

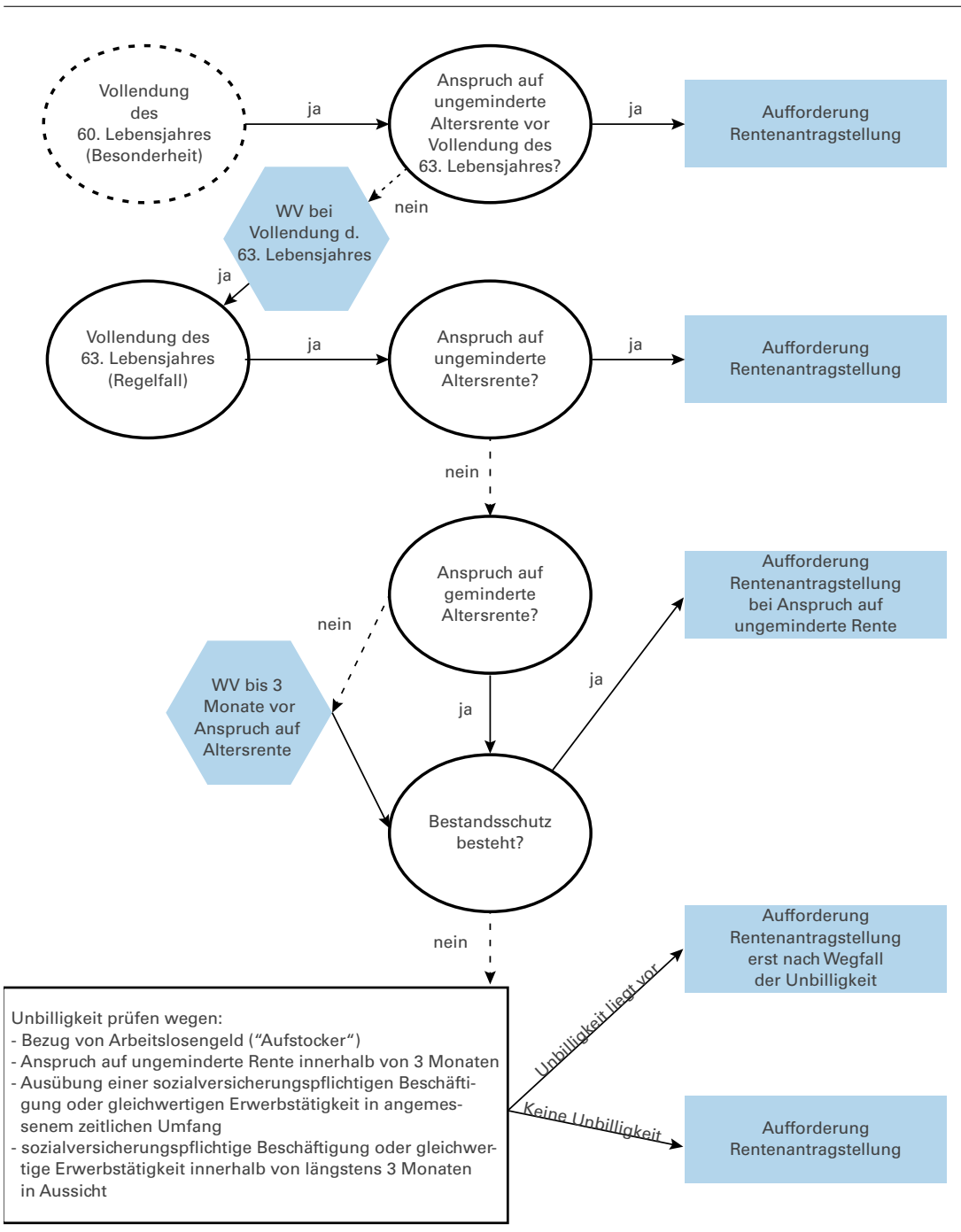

Quelle: Bundesagentur für Arbeit (2013), Anlage 2, vereinfachte und gekürzte Darstellung.

dass die Leistungsbeziehenden ihrerseits unterschiedlich auf die Aufforderung zur Rentenantragstellung reagieren: Einige werden eine Rente dem weiteren ALG-II-Bezug vorziehen, z. B. weil ihre Rente trotz der Abschläge höher ist als die Grundsicherung oder um sich aus der Abhängigkeit vom Jobcenter zu lösen. Andere werden sich in die Pflicht fügen und einen Rentenantrag stellen, und wieder andere werden gegen ihren Willen in die Altersrente genötigt, weil das Jobcenter den Antrag stellt. Entscheidend ist hier, dass eine klare Unterscheidung zwischen Freiwilligkeit und Zwang nicht möglich ist, wenn den Leistungsbeziehenden erst einmal bekannt ist, dass sie zu einer Altersrente nicht nur berechtigt, sondern zu einer Antragstellung sogar verpflichtet sind. Solange die Präferenzen der Leistungsbeziehenden vor und nach der Information über die Pflicht 
zur vorzeitigen Inanspruchnahme einer Altersrente nicht bekannt sind - und sie lassen sich nur durch eine Befragung der Leistungsbeziehenden ermitteln - besteht der Personenkreis der Betroffenen maximal aus vorzeitigen Rentenzugängen aus dem SGB II und minimal aus den Rentenzugängen aufgrund einer stellvertretenden Antragstellung durch die Jobcenter.

Die Größe des „minimalen“ Personenkreises ist nicht bekannt, aber die des „maximalen“ Personenkreises kann näherungsweise bestimmt werden: Dies sind die Beziehenden einer Altersrente, die unmittelbar vor dem Rentenbeginn ALG II erhielten und vorzeitig zwischen der Vollendung des 63. und vor Erreichen der Regelaltersgrenze (bis 2012: 65 Jahre, also zwischen einem und 24 Monaten vorzeitig) in Rente gewechselt sind. Dieser Personenkreis wird einerseits mit abschlagsfreien Rentenzugängen sowie andererseits mit sehr vorzeitigen Rentenzugängen (mehr als 24 Monate) aus dem SGB II verglichen, um abzuschätzen, wie groß das Risiko ist, aufgrund der verpflichtenden Inanspruchnahme einer vorzeitigen Altersrente auch im Alter auf Grundsicherung angewiesen zu sein.

Weiterhin wird der Zusammenhang zwischen Rentenzahlbetrag und Zeitpunkt des Rentenbeginns aus dem ALGII-Bezug heraus für diese drei Gruppen miteinander verglichen. Wenn dieser Zusammenhang für alle drei Gruppen gleich ist oder einem gemeinsamen Trend folgt, so die Überlegung, dann ist der spezifische Einfluss der verpflichtenden Rentenantragstellung für die „Risikogruppe“ geringer einzuschätzen als wenn sich diese Gruppe deutlich von den beiden anderen Gruppen (abschlagsfreie und sehr vorzeitige Rentenzugänge) unterscheidet. Wie SGB-II-Leistungsbezug, Rentenhöhe und Zeitpunkt des Rentenbeginns zusammenhängen, wird zudem im Vergleich mit einer Kontrastgruppe der Renteneintritte aus Altersteilzeitarbeit (ATZ) analysiert. Dies ist als maximaler Kontrast angelegt, da ATZ-Beschäftigte in der Regel stabil beschäftigt sind und ihren Rentenübergang sehr langfristig planen. Denn der Beginn der Altersteilzeitarbeit wird auf den späteren Rentenbeginn abgestimmt. Abschließend werden Überlegungen zur Fürsorgebedürftigkeit im Alter aufgrund abschlagsgeminderter Renten angestellt.

\subsection{Datengrundlage}

Grundlage für die Analysen ist der Scientific Use File Rentenzugang des Jahres 2011, der vom Forschungsdatenzentrum der Rentenversicherung (FDZ-RV) für wissenschaftliche Analysen zur Verfügung gestellt wird. Hierbei handelt es sich um eine $10 \%$-Stichprobe aller Zugänge in Renten (darunter auch Altersrenten) im Jahr 2011. Die Daten beruhen auf Angaben, die die Rentenversicherung für die Durchführung ihrer Aufgaben benötigt, und enthalten neben ausgewählten soziodemografischen Angaben unter anderem Informationen zum Rentenzahlbetrag, zur Zahl der Abschlagsmonate und zum Versicherungsstatus vor
Rentenbeginn. Letzterer wird zum 31.12.2010, also dem letzten Jahreswechsel vor dem Rentenbeginn, erfasst. Die Daten enthalten keine Angaben darüber, ab welchem Zeitpunkt die Bedingungen für einen vorzeitigen Rentenbeginn erstmals erfüllt waren, wer den Rentenantrag gestellt hat (die versicherte Person oder das Jobcenter) und erst recht keine Angabe zum bevorzugten Zeitpunkt des Rentenbeginns durch den Versicherten. Der Datensatz enthält keine Hochrechnungsfaktoren, kann aber in wesentlichen Strukturmerkmalen als repräsentativ angesehen werden.

Es wurden Personen berücksichtigt, die 2011 erstmals eine Altersrente bezogen haben und nicht im Ausland leben. Sie wurden entsprechend des Zeitpunkts des Rentenzugangs in drei Gruppen eingeteilt:

(1) Personen, die abschlagsfrei in Rente gewechselt sind

(2) Personen, die ein bis 24 Monate vorzeitig in Rente gewechselt sind (dies entspricht zumeist einem Altersfenster von 63 bis unter 65 Jahren)

(3) Personen, die mehr als 24 Monate vorzeitig in Rente gewechselt sind.

Darüber hinaus wurden die Personen nach ihrem Versicherungsstatus vor Rentenbeginn in Gruppen eingeteilt. Im Mittelpunkt der Analyse stehen die Personen, die vor Rentenbeginn ALG II bezogen. Als Vergleichsgruppe werden Personen zusammengefasst, die sich vor Rentenbeginn in Altersteilzeitarbeit befanden. ${ }^{4}$ Beide Gruppen umfassen im Datensatz 6.093 (ALG II vor Rente) bzw. 10.397 (ATZ vor Rente) Personen. Angesichts einer Gesamtzahl von Zugängen in Altersrenten mit Wohnsitz in Deutschland im Datensatz von 63.031 Personen entspricht dies 9,7 bzw. 16,5\%. Zahlen der Rentenversicherung berichten - bei einer geringfügig anderen Abgrenzung, die Renten an im Ausland lebende Personen einschließt, - von 8,9 und 15,4\% der Rentenzugänge aus ALG II bzw. Altersteilzeit (Deutsche Rentenversicherung 2013, S. 79).

\subsection{Ergebnisse}

\subsubsection{Häufigkeit des vorzeitigen Rentenzugangs aus ALG II}

Die Risikogruppe, die von der Pflicht zur vorzeitigen Inanspruchnahme einer Altersrente betroffen gewesen sein könnte, sind Personen aus dem ALG II, die ein bis 24 Monate vorzeitig in Rente wechseln. Jeder sechste Rentenzugang aus dem ALG II ist hierzu zu rechnen (Tabelle 2). Bezogen auf die gesamte Untersuchungsstichprobe und

4 Im Datensatz ist kein Fall, der zugleich Altersteilzeit und ALG II aufweist. Ein solcher Fall müsste hier der Altersteilzeit zugeordnet werden, weil er durch die Unbilligkeitsverordnung vor dem verpflichtenden Rentenantrag geschützt ist. 
TABELLE 2

\section{Status vor Rentenbeginn und Zeitpunkt des Rentenbeginns}

Angaben in Prozent

\begin{tabular}{|c|c|c|c|c|}
\hline & ALG II & ATZ* & alle übrigen & insgesamt \\
\hline Ohne Abschläge & 56,9 & 21,6 & 53,4 & 48,5 \\
\hline 1 bis 24 Monate vorzeitig & 16,2 & 28,2 & 19,7 & 20,7 \\
\hline \multirow[t]{2}{*}{25 bis 60 Monate vorzeitig } & 26,9 & 50,3 & 27,0 & 30,8 \\
\hline & 100,0 & 100,1 & 100,1 & 100,0 \\
\hline
\end{tabular}

${ }^{*} \mathrm{ATZ}=$ Altersteilzeit.

Quelle: FDZ-RV - SUFRTZN11XVSBB; Berechnungen des Autors.

Mitteilungen

TABELLE 3

Durchschnittliche monatliche Rentenzahlbeträge und Abschläge

Angaben in Euro

\begin{tabular}{l|c|ccc}
\hline & ALG II & ATZ* & alle übrigen & insgesamt \\
\hline Ohne Abschläge & 423 & 1.218 & 587 & 614 \\
\hline 1 bis 24 Monate vorzeitig & 697 & 1.168 & 914 & 955 \\
\hline 25 bis 60 Monate vorzeitig & 573 & 1.016 & 649 & 741 \\
\hline Alle & 508 & 1.103 & 668 & 724 \\
\hline
\end{tabular}

${ }^{*}$ ATZ $=$ Altersteilzeit

Quelle: FDZ-RV - SUFRTZN11XVSBB; Berechnungen des Autors

Mitteilungen

unter der Annahme, dass diese Teilgruppe entsprechend ihrem Anteil an der Grundgesamtheit vertreten ist, repräsentieren diese $16,2 \%$ ca. 10.000 Menschen. Dieser Wert ist deutlich niedriger als der Wert, der sich aus der dritten Kleinen Anfrage errechnet (für 2011: 15.000 Personen), denn Letzterer bezog sich auf alle Abgänge aus dem ALG II (einschließlich einer unbekannten Zahl von Abgängen in Beschäftigung) nur der 63-Jährigen (und nicht des Altersspektrums von 63 bis unterhalb der Regelaltersgrenze).

Bei der hier zugrunde gelegten Risikogruppe gibt es zwei Arten von Unschärfen: Ein Teil dieser 16\% ist möglicherweise freiwillig in Rente gewechselt (etwa weil die Pflicht individuell noch nicht bekannt oder das 63. Lebensjahr noch nicht vollendet war), und möglicherweise hat ein Teil derjenigen mit höheren Abschlägen (vor Vollendung des 63. Lebensjahres) nach einer Rentenberatung im Jobcenter eine Rente beantragt. Mehr als jeder vierte Rentenzugang aus dem ALG II erfolgte um mehr als zwei Jahre vorzeitig und damit streng genommen noch rascher als das SGB II postuliert. Dies ist ein starkes Indiz dafür, dass die Bedingungen im SGB II (Leistungshöhe, bürokratische Ansprüche, Aktivierungsanspruch) vielfach als so ungünstig bewertet werden, dass viele ALG-II-Beziehende auch um den Preis hoher Abschläge die Grundsicherung verlassen. Daraus kann man aber nicht umgekehrt schließen, dass alle oder mehrheitlich jene, die höchstens zwei Jahre vorzeitig in Rente wechseln, dies gegen ihren Willen tun. Über die Präferenzen ist nichts bekannt. Möglich ist auch, dass die Bedingungen für einen früheren Rentenzugang noch nicht erfüllt waren. Dass längst nicht jede und jeder ALG-II-Beziehende zu einer vorzeitigen Rente berechtigt ist, legt die große Gruppe der abschlagsfreien Rentenzugänge nahe: Über die Hälfte (56,9 \%) ging abschlagsfrei in Altersrente, viele erst mit der Regelaltersgrenze.

Der hohe - im Vergleich mit Rentenzugängen aus ATZ nahezu dreimal so hohe - Anteil der abschlagsfreien Rentenzugänge aus ALG II lässt sich im Jahr 2011 kaum mehr auf den Schutz der 58er-Regelung und aufgrund der Rechts- und Weisungslage wohl auch nicht auf einen Verzicht der Jobcenter zurückführen, den Vorrang der Rente zu erwirken. Vielmehr sind die sehr niedrigen Rentenzahlbeträge (Tabelle 3) gerade in dieser Gruppe ein Indiz dafür, dass die - auf den gesamten Lebenslauf betrachtete - Erwerbsbeteiligung in vielen Fällen wohl schwach war und deshalb eine vorzeitig beziehbare Rente nicht infrage kommt (Wübbeke 2007). Der durchschnittliche monatliche Rentenzahlbetrag lag bei $423 €$. Wohl wegen der stärkeren Erwerbsteilhabe über den Lebenslauf hinweg sind nicht nur die Rentenzahlbeträge der übrigen Rentenzugänge aus ALG II höher, sondern überhaupt erst die Bedingungen für eine der vorzeitig beziehbaren Altersrenten erfüllt, die durchweg mehr als die Mindestversicherungszeit von fünf Jahren voraussetzen (Über- 
Relative durchschnittliche Rentenzahlbeträge und Abschläge, nach Geschlecht und Region

Angaben in Prozent* und in absoluten Zahlen

\begin{tabular}{|c|c|c|c|c|c|c|c|c|}
\hline & \multicolumn{4}{|c|}{ ALG II } & \multicolumn{4}{|c|}{ ATZ** } \\
\hline & \multicolumn{2}{|c|}{ Männer } & \multicolumn{2}{|c|}{ Frauen } & \multicolumn{2}{|c|}{ Männer } & \multicolumn{2}{|c|}{ Frauen } \\
\hline & West & Ost & West & Ost & West & Ost & West & Ost \\
\hline ohne Abschläge (in \%) & 85,1 & 89,1 & 83,8 & 93,7 & 107,1 & 113,0 & 111,7 & 115,7 \\
\hline 1 bis 24 Monate (in \%) & 135,8 & 113,8 & 150,8 & 117,1 & 104,1 & 102,4 & 102,4 & 105,5 \\
\hline 25 bis 60 Monate (in \%) & 121,8 & 99,4 & 128,5 & 98,7 & 93,7 & 91,0 & 94,3 & 93,7 \\
\hline Durchschn. Rentenbetrag (Euro) & 556 & 650 & 390 & 520 & 1.304 & 1.106 & 865 & 940 \\
\hline
\end{tabular}

* in Prozent des durchschnittlichen Rentenzahlbetrages der jeweiligen Gruppe. Lesebeispiel: Männer aus Westdeutschland, die eine Altersrente aus ALG II kommend ohne Abschläge beginnen, erhalten im Durchschnitt 85,1 \% des durchschnittlichen Rentenzahlbetrages aller männlichen westdeutschen Rentenzugänge aus ALG II, welcher $556 €$ beträgt (also ca. $473 €$ ).

${ }^{*}$ *ATZ $=$ Altersteilzeit.

Quelle: FDZ-RV - SUFRTZN11XVSBB; Berechnungen des Autors.

sicht 1). Der Unterschied zu den Rentenzugängen aus ATZ ist besonders ausgeprägt. ATZ-Beschäftigte dürften beinahe ausnahmslos die Bedingungen für eine vorzeitig beziehbare Rente erfüllen. Der durchschnittliche Rentenzahlbetrag ist mehr als doppelt so hoch wie der der Rentenzugänge aus ALG II. Und er ist umso höher, je weniger Abschläge fällig wurden (und je länger aufgrund des fortbestehenden Beschäftigungsverhältnisses Beiträge gezahlt wurden).

Der Unterschied im Rentenzugangsverhalten und die Besonderheit der ALG-II-Beziehenden bestätigt sich, wenn man untersucht, wie Rentenzahlbetrag und Rentenzugangszeitpunkt zusammenhängen und dabei berücksichtigt, dass die beiden Gruppen der ALG-II-Beziehenden und der Altersteilzeitbeschäftigten hinsichtlich des Frauenanteils sowie des Anteils von West- bzw. Ostdeutschen unterschiedlich zusammengesetzt sind (Tabelle 4).

Auch in einer - hier nicht dargestellten ${ }^{5}$ - multinomialen Regressionsschätzung bestätigt sich, dass Frauen und Ostdeutsche ceteris paribus mit größeren Abschlägen in Rente wechseln (so auch statt vieler: Radl 2007, S. 54ff.). Weiterhin bestätigt sich der oben dargestellte Befund, dass Altersteilzeitbeschäftigte ceteris paribus häufiger vorzeitig in Rente gehen (bezogen auf die Referenzgruppe aller übrigen Zugänge in Altersrente) und ALG-II-Beziehende seltener bzw. später. Insbesondere bestätigt sich der Befund, dass unter den Rentenzugängen aus ALG II der höchste durchschnittliche Rentenzahlbetrag aus der Gruppe derjenigen kommt, die direkt der verpflichtenden Inanspruchnahme einer Altersrente unterliegen (1-24 Monate Abschläge), der zweithöchste aus der Gruppe mit noch höheren Abschlägen und der niedrigste aus der Gruppe, die abschlagsfrei in Altersrente kommt. Bei Rentenzugängen aus Altersteilzeit ist der Rentenzahlbetrag umso höher, je niedriger die Abschläge sind und je später der Rentenzugang erfolgt.
Der Befund für die ALG-II-Beziehenden lässt sich nicht eindeutig interpretieren. Er kann bedeuten, dass sie insbesondere dann in Rente „flüchten“, wenn ihre Rente höher ist als das ALG II. Er kann aber auch bedeuten, dass viele den frühestmöglichen (oder einen sehr frühen) Zeitpunkt für den Rentenbeginn nutzen, und dieser Zeitpunkt liegt bei langen Beitragszeiten früher als bei einer - unter ALG-II-Beziehenden verbreiteten - schwachen Arbeitsmarktbindung bzw. kurzen Beitragszeiten. Wahrscheinlich spielen für den Rentenbeginn auch die Beratung im Jobcenter und der Hinweis auf die Pflicht zur Inanspruchnahme einer vorzeitigen Altersrente eine Rolle. Dies scheint aber nur ein möglicher Einfluss neben den beiden starken Treibern des Rentenzugangs für ALG-II-Beziehende, den rentenrechtlichen Voraussetzungen und dem Rentenzahlbetrag zu sein.

\subsubsection{Grundsicherungsrisiko aufgrund der Abschläge?}

Wie hoch ist das Risiko, aufgrund der Pflicht, ab dem 63. Lebensjahr die Rente zu beanspruchen, nach Vollendung der Regelaltersgrenze auf Grundsicherung im Alter angewiesen zu sein? Diese Frage lässt sich hier nicht abschließend beantworten, da die Rente eine individuelle Anspruchsleistung ist, die Fürsorgebedürftigkeit aber vom Gesamteinkommen und der Vermögenslage des Haushaltes (und unter Umständen vom Einkommen der Kinder) abhängt. Folgende Überlegungen lassen sich aber anstellen: Solange die Rentenzugänge aus dem SGB II Arbeitslosengeld II bezogen haben, waren sie bedürftig. Wenn der Rentenzahlbetrag nicht höher ist als die Grundsicherungsleistung (einschließlich der Kosten für Unterkunft

5 Die Ergebnisse können beim Autor angefordert werden. 
und Heizung) und keine weiteren Einkommen hinzutreten, dann hat sich die materielle Gesamtsituation nicht geändert. Die „Bedürftigkeit“ im Sinne einer Abhängigkeit von Sozialleistungen hat sich durch den Rentenbezug verringert, ist aber nicht vollständig aufgehoben.

Die Daten enthalten keinen Hinweis, ob mit dem Rentenbezug weitere Einkommen (Betriebsrente oder private Altersvorsorge) hinzutreten, die zuvor nicht bezogen werden konnten. Ergebnisse der Studie „Altersvorsorge in Deutschland (AVID) “ deuten an, dass die zweite und dritte Schicht der Alterssicherung einen ungleichheitsverstärkenden Effekt haben (Nürnberger 2007; Frommert/ Himmelreicher 2012). Möglicherweise entgehen einige Niedrigrentenbeziehende gerade durch betriebliche und private Alterssicherung der Fürsorgebedürftigkeit, doch kann man nicht davon ausgehen, dass gerade die Niedrigrentenbeziehenden durch ihre Ansprüche an die zweite und dritte Schicht vor einem Grundsicherungsanspruch im Alter geschützt sind.

Der durchschnittliche Zahlbetrag im SGB II für Alleinstehende betrug im Jahr $2011716 €$ pro Monat und lag in anderen Haushaltskonstellationen niedriger. Dies entspricht fast exakt dem durchschnittlichen Rentenzahlbetrag der "Risikogruppe“ (Renteneintritt aus ALG II mit ein bis 24 Abschlagsmonaten), der bei $697 €$ lag (Tabelle 3). Zwar handelt es sich hier nur um Durchschnittswerte, doch es ist ohne Weiteres vorstellbar, dass es gerade die Abschläge sind, die einen Rentenzahlbetrag unterhalb des bisherigen Grundsicherungsanspruchs bewirken. Immerhin betragen die Abschläge maximal 7,2\%, was, bezogen auf den durchschnittlichen Rentenzahlbetrag, einer ungekürzten - vor Abschlägen ermittelten - Rente von $747 €$ entspricht. Es ist zu betonen, dass zusätzliche individuelle Alterseinkommen hier nicht berücksichtigt sind und die tatsächlichen Haushaltskonstellationen, ALG-II-Ansprüche und Rentenzahlbeträge nicht bekannt sind. Doch die hier angestellten Überlegungen zeigen, dass Fürsorgebedürftigkeit aufgrund gekürzter Renten nicht nur in Einzelfällen zu vermuten ist. Es ist allerdings auch darauf hinzuweisen, dass den hier präsentierten Ergebnissen zufolge das Risiko eines Fürsorgebedarfs im Alter deutlich höher ist bei jenen Personen, die abschlagsfrei aus ALG II in Altersrente wechseln und sogar auch höher ist bei Personen, die - aufgrund der Bestimmungen des SGB II nicht zwingend - mit noch höheren Abschlägen aus dem ALG II heraus eine Rente beantragen.

\section{Diskussion und Fazit}

Den Ergebnissen dieser Untersuchung zufolge stellen sich Rentenzahlbeträge und rentenrechtliche Zugangsvoraussetzungen als die starken Einflussfaktoren für den Rentenzugang aus dem ALG-II-Bezug heraus dar. Die Gruppe von Personen, die für eine verpflichtende Rentenantragstellung infrage kommt, ist deutlich kleiner als die Gruppe jener, die abschlagsfrei (überwiegend an der Regelaltersgrenze und vermutlich aufgrund fehlender versicherungsrechtlicher Voraussetzungen für eine vorzeitig beziehbare Altersrente) bzw. zum frühestmöglichen Zeitpunkt gegebenenfalls auch vor Vollendung des 63. Lebensjahres eine Rente bezieht. Gleichwohl deuten die durchschnittlichen Rentenzahlbeträge darauf hin, dass ein Grundsicherungsbezug im Alter aufgrund des durch den vorzeitigen Rentenbezug geminderten Rentenanspruchs nicht nur Einzelfälle abbildet. Diese Vermutung ergibt sich nicht allein aus dem durchschnittlichen Rentenzahlbetrag, sondern auch aus der Tatsache, dass im ALG II die Personen bereits bedürftig sind, und deshalb vielfach neben der Rente weitere Alterseinkünfte erforderlich sind, um die Grundsicherungsschwelle zu überschreiten. Unbestritten gibt es das Risiko, aufgrund des vorzeitigen Rentenbezugs - der wiederum nur zum Teil auf die Verpflichtung im SGB II und das Wirken der Jobcenter, eine vorzeitige Rente zu beantragen, zurückzuführen ist - fürsorgebedürftig im Alter zu werden. Quantitativ viel größer aber ist ein anderes Risiko: nämlich dass die große Gruppe der ALG-II-Beziehenden, die zwar abschlagsfrei, aber mit sehr niedrigen Rentenansprüchen in die Altersrente wechselt, trotz des „regulären“ Rentenbezugs grundsicherungsbedürftig ist.

Umfangreichere Auswertungen mit den vorhandenen Daten sowie zusätzliche Daten können die hier vorgelegten Ergebnisse noch einmal deutlich differenzieren und möglicherweise auch revidieren. Von Interesse ist insbesondere eine genauere quantitative Abschätzung des betroffenen Personenkreises im Zeitverlauf. Im Prinzip liegen aussagekräftige Daten zur Rentenberatung in den Jobcentern vor, können aber aktuell nicht genutzt werden. Alternativ würde eine Befragung von rentennahen Personen weiterführenden Aufschluss geben. Sie könnte insbesondere geeignet sein, das Wechselspiel von Informationsgrundlagen, Präferenzen, Freiwilligkeit und Zwang im Altersübergang zu untersuchen.

Angesichts der Tatsache, dass viele ALG-II-Beziehende zum frühestmöglichen Rentenzeitpunkt in Altersrente wechseln und noch mehr ALG-II-Beziehende gar nicht vorzeitig in Rente gehen können, drängt sich die Vermutung auf, dass sich am Renteneintrittsverhalten aus dem ALG II heraus nicht viel ändern würde, wenn es die Verpflichtung zur Inanspruchnahme einer Altersrente nicht geben würde. Wohl aber würden sich das Rentenzugangsverhalten und die Fürsorgebedürftigkeit im Alter von rentennahen ALG-II-Beziehenden ändern, wenn sie konsequent in Beschäftigung vermittelt werden würden. Eine Abschaffung der verpflichtenden Inanspruchnahme von vorzeitig beziehbaren Altersrenten würde die Voraussetzungen verbessern, ältere ALG-II-Beziehende in Beschäftigung zu vermitteln, weil Jobcenter dann von bürokratischem Aufwand entlastet werden, der nicht ihrem Kerngeschäft entspricht. 


\section{LITERATUR}

Birkwald, M. (2013): Welle von Zwangsverrentungen droht. Bundesregierung legt widerwillig erste Zahlen vor, Berlin

Brussig, M./Wübbeke, C. (2009): Policy-making in ageing labour markets: The case of hidden early retirement in Germany, in: Kuhn, M./Ochsen, C. (Hrsg.): Labour markets and demographic change, Wiesbaden, S. 252-280

BT-Drucksache 16/13346: Antwort der Bundesregierung auf die Kleine Anfrage der Abgeordneten Klaus Ernst u. a. - Zwangsverrentungen von SGB-II-Beziehenden und Folgen der Abschaffung der sogenannten 58er-Regelung, Berlin BT-Drucksache 17/5410: Antwort der Bundesregierung auf die Kleine Anfrage der Abgeordneten Klaus Ernst, Matthias W. Birkwald, Diana Golze, weiterer Abgeordneter und der Fraktion DIE LINKE, Berlin

BT-Drucksache 18/152: Antwort der Bundesregierung auf die Kleine Anfrage der Abgeordneten Matthias W. Birkwald u. a. - Drucksache 18/33 - Zwangsver rentungen von Leistungsberechtigten nach dem Zweiten Buch Sozialgesetzbuch (2014), Berlin

BT-Drucksache 18/152+681: Antwort der Bundesregierung auf die Kleine Anfrage der Fraktion DIE LINKE - Zwangsverrentungen von Leistungsberechtigten nach dem Zweiten Buch Sozialgesetzbuch, Berlin

Bundesagentur für Arbeit (2013): Zweites Buch Sozialgesetzbuch - SGB II.

Fachliche Hinweise. § 12a SGB II. Vorrangige Leistungen

Deutsche Rentenversicherung (2013): Rentenversicherung in Zeitreihen, Oktober, DRV-Schriften (22), Berlin

Frommert, D./Himmelreicher, R. (2012): Entwicklung und Zusammensetzung von Alterseinkünften in Deutschland, in: Vogel, C./Motel-Klingebiel, A. (Hrsg.): Altern im Sozialen Wandel. Die Rückkehr der Altersarmut?, Wiesbaden,

S. $141-159$

Mika, T./Lange, J./Stegmann, M. (2014): Erwerbsminderungsrenten nach Bezug von ALG II: Auswirkungen der Reformen auf die Versicherungsbiografien, in: WSI-Mitteilungen 67 (4), S. 277-285, http://www.boeckler.de/wsi-mitteilungen_47210_47221.htm

Nürnberger, I. (2007): Was bekommen künftige Rentner? Neue AVID-Studie liefert wichtige Trendaussagen, in: Soziale Sicherheit 56 (12), S. 405-416

Radl, J. (2007): Individuelle Determinanten des Renteneintrittsalters. Eine empirische Analyse von Übergängen in den Ruhestand, in: Zeitschrift für Soziolo gie 36 (1), S. 43-64

Schmidt, M. (2007): Das politische System Deutschlands. Institutionen, Willensbildung und Politikfelder: Bundeszentrale für Politische Bildung: Schriftenreihe Bundeszentrale für Politische Bildung, Bd. 600, Bonn

Soziologisches Forschungsinstitut (SOFI)/Institut für Arbeit und Qualifikation (IAQ)/Forschungsinstitut Internationaler Arbeitsmarkt (FIA) (2013): Beratungs konzeption SGB II-Pilotierung. Evaluationsbericht, Göttingen/Duisburg/Berlin Wübbeke, C. (2007): Ältere Bezieher von Arbeitslosengeld II: Einmal arm, immer arm?, IAB-Kurzbericht 14/2007, Nürnberg

Wübbeke, C. (2013): Ältere Arbeitslose am Scheideweg zwischen Erwerbsleben und Ruhestand: Gründe für ihren Rückzug vom Arbeitsmarkt, in: Journal for Labour Market Research 46 (1), S. 61-82

\section{AUTOR}

MARTIN BRUSSIG, PD Dr., arbeitet als Wissenschaftler am Institut Arbeit und Qualifikation (IAQ) an der Universität Duisburg-Essen. Arbeitsschwerpunkte: Alterserwerbstätigkeit, Arbeitsmarktpolitik.

@ martin.brussig@uni-due.de 